\title{
Special considerations of breast cancer management in the elderly
}

\author{
Lauren F Cornell*,1 (D), Sarah A Mclaughlin¹, Sandhya Pruthi² \& Dawn M Mussallem \\ ${ }^{1}$ Jacoby Center for Breast Health, Mayo Clinic, Jacksonville, FL 32224, USA \\ ${ }^{2}$ Breast Diagnostic Clinic, Mayo Clinic, Rochester, MN 55905, USA \\ *Author for correspondence: cornell.lauren@mayo.edu
}

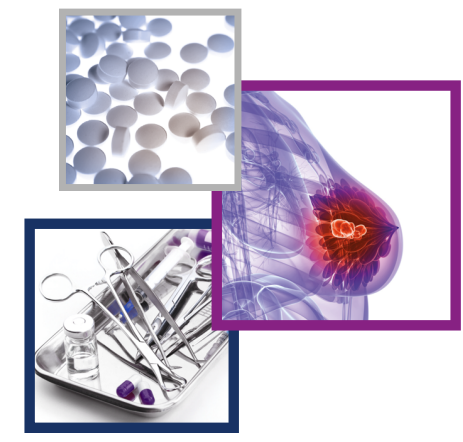

\section{Practice points}

- There are limited clinical trial data specific to elderly patients with breast cancer and treatment recommendations are generally made utilizing extrapolated data.

- In addition to considering standard guidelines, management of elderly patients needs to be tailored to patient functional status, individual tumor biology and presence of competing comorbidities.

- Surgical excision for primary breast malignancy is considered generally safe and should be recommended for most patients, regardless of age.

- Endocrine therapy should be offered to patients with hormone receptor positive disease, taking into account medication risks and underlying comorbidities.

- The role for axillary surgery, breast radiation and chemotherapy should be individualized in elderly patients with utilization of informed shared-decision making.

There are increasing numbers of elderly patients diagnosed with breast cancer. These patients are underrepresented in available clinical trials, and as such, there are limited evidence-based guidelines for treatment in this population. Elderly patients have unique needs and management strategies should be tailored accordingly. This article reviews available literature regarding breast cancer management and special considerations in elderly patients.

First draft submitted: 30 April 2020; Accepted for publication: 17 July 2020; Published online: 1 September 2020

Keywords: breast cancer $\bullet$ elderly $\bullet$ geriatric

Due to increased life expectancy and growth of older population groups, the USA is experiencing an aging population. By 2030, 20\% of the US population will be older than 65 years of age. Patients diagnosed with breast cancer (BC) are no exception. In fact, nearly $50 \%$ of patients diagnosed with BC in the US are over 65-years old [1].

Successful cancer care seeks to minimize treatment toxicity, enhance quality of life, reduce cancer recurrence and improve survival. In elderly patients, this balance is complicated by increased frailty, competing comorbidities and shorter life expectancy. While age alone does not define a patient's goals of care, certain considerations should be taken into account when counseling older patients prior to beginning therapy. Clinicians need to consider baseline functional status, the patient's cancer type and risk for recurrence, risk for non-BC related death and the risk for toxicity of each individual treatment being considered. Patient preferences and values should also be considered. Although this is true for any patient, elderly patients likely have different priorities than their younger BC counterparts.

Historically, older patients were underrepresented and often excluded from most large randomized clinical trials resulting in a lack of evidence-based treatment recommendations for this population. Recently this issue has been recognized, although the literature specific to the elderly population remains limited.

Herein, we will provide a concise review of the available literature and data specific to the treatment of elderly patients with BC.

Future Medicine 


\section{Surgery}

In discussing surgical options with patients it is important to keep in mind that breast surgery is considered to have an intrinsically low cardiac risk [2]. For all BC patients, surgery related mortality from mastectomy or lumpectomy is very low, ranging from 0 to $0.3 \%$ [3]. In many cases breast surgery can be performed with local/regional anesthesia only, avoiding use of general anesthesia. Still the question arises whether certain elderly patients can avoid surgery altogether.

The Group for Research on Endocrine Therapy in the Elderly (GRETA) trial was a multicenter randomized controlled trial designed to compare surgery with tamoxifen to primary endocrine therapy with tamoxifen alone. Primary endocrine therapy with tamoxifen resulted in a significantly higher rate of local disease progression and nonsignificant increase in mortality [4].

Similar results were seen in a meta-analysis of seven studies comparing primary endocrine therapy with surgery plus endocrine therapy in women over 70 years of age, deemed fit for surgery. There was a significant difference in progression free survival (hazard ratio [HR]: $0.65 ; 95 \% \mathrm{CI}: 0.53-0.81 ; \mathrm{p}=0.0001$ ), although no significant difference was seen in overall survival (OS) (HR: 0.86; 95\% CI: 0.73-1.00; $\mathrm{p}=0.06$ ) [5]. This was regardless of the type of breast surgery performed (breast conservation or mastectomy).

No formal quality of life assessments were performed in any of the trials included in this meta-analysis. One study did suggest increased psychosocial morbidity in patients undergoing surgery at 3 months postoperatively, but this had resolved by 2 years [6].

Of note, most of the data comparing surgery with endocrine therapy alone utilized tamoxifen. Aromatase inhibitors (AIs) have now been studied in both the adjuvant and neoadjuvant settings, and have proven to be superior to tamoxifen in achieving local tumor response [7].

In one review of patients receiving primary endocrine therapy with either tamoxifen (55\%) or AI (45\%) without surgery, $58 \%$ of patients experienced an initial response; however, at mean follow-up of 2.6 years, $35 \%$ of patients had already displayed progression, with average time to progression 20 months [8]. Based on this cumulative data, in the absence of significant comorbidities, breast surgery should be considered in most BC patients, regardless of age.

Surgical evaluation of the axilla is not necessarily recommended for all elderly patients. When considering axillary lymph node evaluation for an older patient, two questions should be considered: what is the expected morbidity of the procedure, and how will the results affect management and BC specific outcomes for the patient.

In regards to morbidity, one large longitudinal study of BC patients over 67 years of age showed that women who underwent axillary surgery were three-times more likely to develop arm problems including lymphedema, parasthesias, arm pain, weakness or impaired shoulder function [9]. Another randomized trial showed that avoidance of axillary surgery resulted in improved quality of life for this older population of patients, especially in the early postoperative period [10].

For early stage, clinically node-negative elderly BC patients, the likelihood of detecting pathologically positive lymph nodes that will impact clinical outcomes is considered low. In one large prospective study by Martelli et al. women over 70-year old with operable BC without palpable axillary lymphadenopathy were offered breast surgery without axillary evaluation or radiation therapy. At 15 -year follow-up, only $4.2 \%$ of women were found to have detectable axillary disease. A large portion passed away within 15 years, with $83 \%$ of deaths unrelated to their $\mathrm{BC}$ [11]. The authors concluded that these patients may be safely treated without axillary dissection due to the low cumulative incidence of axillary disease.

In 2016, the Society of Surgical Oncology paired with the American Board on Internal Medicine to launch the Choosing Wisely campaign. These guidelines formally recommend against routine use of sentinel lymph node evaluation in patients over 70 years of age with clinically node negative, hormone receptor positive, HER2-negative early stage BC [12].

\section{Radiation therapy}

For all women with $\mathrm{BC}$, postoperative radiation therapy following breast conserving surgery has been shown to substantially reduce the risk for ipsilateral BC recurrence although with lesser established impact on survival [13]. The Cancer and Leukemia Group B (CALGB) 9343 trial was a large, randomized clinical trial designed specifically to assess the added benefit of adjuvant radiation therapy in women older than 70 years of age with estrogen receptor positive, clinically stage I tumors [14]. At 5-year follow-up, women who received adjuvant radiation therapy had significantly lower risk for ipsilateral recurrence, although the absolute risk was low in both groups (4 vs 
$1 \% ; \mathrm{p}=0.001)$. As expected, there was no difference in OS. These findings held true at 12.6-year follow-up, where risk for recurrence without radiation therapy was approximately 10 versus $2 \%$ in those who received radiation. No differences were seen in time to mastectomy, time to distant metastasis, BC-specific survival, or OS between the two groups [15]. Similar results were seen in the PRIME-II trial which included women 65 years of age or older with perceived low risk tumors (hormone receptor positive, $<3 \mathrm{~cm}$ ) [16].

Based on this data, it is reasonable to offer omission of radiation to older BC patients with hormone receptor positive early stage $\mathrm{BC}$, especially in the presence of competing comorbidities. However, it is also important to note, these trials looked at patients who received endocrine therapy for at least 5 years. In patients where there is a concern for medication compliance or poor tolerability, they need to be advised that risk for recurrence would be substantially higher [17]. Generally, it is accepted that patients with more aggressive tumor subtypes and advanced disease benefit more from the use of radiation therapy, regardless of age.

If radiation therapy is recommended, more tolerable courses, including partial breast irradiation or hypofractionated schedules, may be considered as an appropriate option for older patients [18].

\section{Chemotherapy}

Chemotherapy is known to improve BC specific outcomes and OS in women with BC. However, chemotherapy related toxicity is important to consider, specifically in older patients where underlying comorbidities and shorter life expectancy play a larger role. In a review of the SEER database of women aged 65 years of age and older with stage I-III BC between 1991 and 1999, approximately 10\% received chemotherapy. Of those, a significant reduction was noted in $\mathrm{BC}$ mortality for women with lymph node positive, estrogen receptor negative BC (HR: 0.72; 95\% CI: 0.54-0.96). Chemotherapy did not improve survival in women with estrogen receptor positive BC, regardless of lymph nodal status [19].

In the Cancer and Leukemia Group B (CALGB) 49907 randomized clinical trial, 633 patients over 65 years of age with stage I-IIIB BC were assigned to standard chemotherapy with anthracycline based regimen versus capecitabine alone. All patients had more than 5-year life expectancy, without competing comorbidity. At a median follow-up of 2.4 years, patients assigned to capecitabine were twice as likely to have a relapse and almost twice more likely to die than patients assigned to standard chemotherapy [20]. More recently, these results were updated with 11-year follow-up and continued to show inferiority of capecitabine in regards to recurrence risk and BC specific mortality, although OS rates were not statistically different. In a subset analysis, the benefit of standard chemotherapy was significantly more pronounced in those patients with hormone receptor negative disease. In fact, there was no difference in recurrence risk among hormone receptor positive patients [21].

A substudy of 350 patients from the CALGB 49907 cohort found that capecitabine was associated with better quality of life during treatment; however, this was similar between the two groups by 1 year [22]. Patients receiving standard chemotherapy were nearly twice as likely to experience moderate to severe toxic effects compared with those on capecitabine (64 vs 33\%). Hematologic grade 3-4 toxic effects were experienced in more than 50\% of patients who received chemotherapy but only $2 \%$ receiving capecitabine [20]. The increased risk for toxicity seen in older patients may be attributed to poor bone marrow reserve, reduced functional status and decreased renal clearance. Another retrospective review of three large, randomized clinical trials found that patients over 65 years of age experienced higher hematologic toxicity from chemotherapy, although this did not result in increased mortality compared with younger patients [23].

Genomic testing has emerged in recent years as a method to determine predictive information of chemotherapy benefit in specific patients with early stage hormone receptor positive BC. However, most genomic assays, including the 21-gene recurrence score (Oncotype DX), have not been well validated in older patients. One study demonstrated that women aged over 70 years of age were significantly less likely to undergo genomic testing, but when were tested, they had a similar distribution of Oncotype DX recurrence scores compared with younger patients [24].

The ability to predict which patients will experience chemotherapy related toxicity may allow for more individualized treatment recommendations. Hurria et al. utilized a prospective study design of older patients undergoing chemotherapy to create a predictive model for development of toxicity. Factors considered include geriatric assessment variables, laboratory test values and patient/tumor characteristics. A scoring system was created based on these variables to classify patients as low (30\% risk), intermediate (52\% risk) or high ( $83 \%$ risk) risk for chemotherapy related grade 3-5 toxicity [25]. Calculating and discussing this risk with patients may be useful in guiding their decisions for systemic therapy. 


\section{Endocrine therapy}

The use of endocrine therapy in patients with hormone receptor positive disease is generally recommended, regardless of age. Both tamoxifen and the AIs may be used in elderly patients, similar to younger patients. There is well established evidence for improvement in BC related outcomes with limited toxicity $[7,26]$. Extensive data has demonstrated AIs are superior to tamoxifen in the adjuvant and neoadjuvant settings, and this benefit may be even greater in women over 65 years of age [7,27].

Patients should be counseled regarding potential medication risks and side effects, taking into account underlying comorbidities which may impact these risks. Tamoxifen is associated with an increased risk of endometrial cancer, and in women with an intact uterus, an AI would be preferred. However, AIs are associated with an increased risk of bone thinning and in patients with osteoporosis, tamoxifen may be preferred as it has been shown to improve bone density [28].

\section{Conclusion}

As the population ages, we will treat more elderly patients with BC. More clinical trial data in elderly patients is needed to fully understand risk to benefit ratios for this population. In 2008 the NCCN Task Force on BC in the older woman was created to provide consensus on management for these patients. Within this, there was agreement in regards to well-established benefit of curative surgery and endocrine therapy in older patients but continued areas of controversy were acknowledged in regards to role for radiation, chemotherapy and axillary surgery [29]. Management strategies should be individualized with utilization of shared-decision making based on a patient's diagnosis, functional status, preferences and values.

Geriatric prognostic indices may be utilized by providers to aid in this shared decision making. A comprehensive geriatric assessment can be used to provide a range of data to predict morbidity/mortality, although clinical use is often limited due to the time required for these assessments and expertise needed to interpret results [29]. The University of California San Francisco designed a repository of published geriatric prognostic indices, ePrognosis [30,31]. This is intended for use by clinicians to provide additional information on possible outcomes and prognoses for elderly patients when counseling on individualized management strategies.

\section{Future perspective}

Optimal management for elderly patients with BC remains poorly defined, with limited evidence-based recommendations. It is unlikely large, prospective randomized trials focused on women over 70 years of age will be feasible given the small number of $\mathrm{BC}$ specific events expected and competing mortality from other age related medical conditions. Treatment recommendations should be based on best available extrapolated data tailored to patient functional status, individual tumor biology and presence of competing comorbidities. Multidisciplinary input aids in these discussions.

Financial \& competing interests disclosure

The authors have no relevant affiliations or financial involvement with any organization or entity with a financial interest in or financial conflict with the subject matter or materials discussed in the manuscript. This includes employment, consultancies, honoraria, stock ownership or options, expert testimony, grants or patents received or pending, or royalties.

No writing assistance was utilized in the production of this manuscript.

Open access

This work is licensed under the Attribution-NonCommercial-NoDerivatives 4.0 Unported License. To view a copy of this license, visit http://creativecommons.org/licenses/by-nc-nd/4.0/

\section{References}

Papers of special note have been highlighted as: $\bullet$ of interest

1. National Cancer Institute. Cancer stat facts: female breast cancer (2020). https://seer.cancer.gov/statfacts/html/breast.html

2. Eagle KA, Berger PB, Calkins $\mathrm{H}$ et al. ACC/AHA guideline update for perioperative cardiovascular evaluation for noncardiac surgery - executive summary a report of the American College of Cardiology/American Heart Association task force on practice guidelines (committee to update the 1996 guidelines on perioperative cardiovascular evaluation for noncardiac surgery). Circulation 105(10), 1257-1267 (2002).

3. Wildiers H, Kunkler I, Biganzoli L et al. Management of breast cancer in elderly individuals: recommendations of the International Society of Geriatric Oncology. Lancet Oncol. 8(12), 1101-1115 (2007). 
4. Mustacchi G, Ceccherini R, Milani S et al. Tamoxifen alone versus adjuvant tamoxifen for operable breast cancer of the elderly: long-term results of the Phase III randomized controlled multicenter GRETA trial. Ann. Oncol. 14(3), 414-420 (2003).

5. Hind D, Wyld L, Reed MW. Surgery, with or without tamoxifen, vs tamoxifen alone for older women with operable breast cancer: cochrane review. Br. J. Cancer 96(7), 1025-1029 (2007).

6. Fallowfield F. Quality of life in the elderly woman with breast cancer treated with tamoxifen and surgery or tamoxifen alone. J. Womens Health 3(1), 17-20 (1994).

7. Early Breast Cancer Trialists' Collaborative G. Aromatase inhibitors versus tamoxifen in early breast cancer: patient-level meta-analysis of the randomised trials. Lancet 386(10001), 1341-1352 (2015).

8. Wink CJ, Woensdregt K, Nieuwenhuijzen GA et al. Hormone treatment without surgery for patients aged 75 years or older with operable breast cancer. Ann. Surg. Oncol. 19(4), 1185-1191 (2012).

9. Mandelblatt JS, Edge SB, Meropol NJ et al. Sequelae of axillary lymph node dissection in older women with stage 1 and 2 breast carcinoma. Cancer 95(12), 2445-2454 (2002).

10. International Breast Cancer Study G et al.International Breast Cancer Study G, Rudenstam CM, Zahrieh D et al. Randomized trial comparing axillary clearance versus no axillary clearance in older patients with breast cancer: first results of International Breast Cancer Study Group Trial 10-93. J. Clin. Oncol. 24(3), 337-344 (2006).

11. Martelli G, Miceli R, Costa A et al. Elderly breast cancer patients treated by conservative surgery alone plus adjuvant tamoxifen: fifteen-year results of a prospective study. Cancer 112(3), 481-488 (2008).

12. Choosing Wisely Campaign. Society of Surgical Oncology (2019). www.choosingwisely.org/clinician-lists/sso-sentinel-node-biopsy-in-node-negative-women-70-and-over/

- Considers practice changing in regards to management of the axilla in older patients with breast cancer.

13. Darcy S, McGale P, Correa C et al. Effect of radiotherapy after breast-conserving surgery on 10-year recurrence and 15 -year breast cancer death: meta-analysis of individual patient data for 10,801 women in 17 randomised trials. Lancet 378(9804), 1707-1716 (2011).

14. Hughes KS, Schnaper LA, Berry D et al. Lumpectomy plus tamoxifen with or without irradiation in women 70 years of age or older with early breast cancer. N. Engl. J. Med. 351(10), 971-977 (2004).

15. Hughes KS, Schnaper LA, Bellon JR et al. Lumpectomy plus tamoxifen with or without irradiation in women age 70 years or older with early breast cancer: long-term follow-up of CALGB 9343. J. Clin. Oncol. 31(19), 2382-2387 (2013).

- Results from this trial may significantly impact management decisions and counseling of elderly patients in regards to the role for adjuvant radiation therapy.

16. Kunkler IH, Williams LJ, Jack WJ, Cameron DA, Dixon JM. Breast-conserving surgery with or without irradiation in women aged 65 years or older with early breast cancer (PRIME II): a randomised controlled trial. Lancet Oncol. 16(3), 266-273 (2015).

17. Blamey RW, Bates T, Chetty U et al. Radiotherapy or tamoxifen after conserving surgery for breast cancers of excellent prognosis: British Association of Surgical Oncology (BASO) II trial. Eur. J. Cancer 49(10), 2294-2302 (2013).

18. Giugliano FM, Falivene S, Esposito E et al. External radiotherapy for breast cancer in the elderly. Aging Clin. Exp. Res. 29(Suppl. 1), 149-157 (2017).

19. Giordano SH, Duan Z, Kuo YF, Hortobagyi GN, Goodwin JS. Use and outcomes of adjuvant chemotherapy in older women with breast cancer. J. Clin. Oncol. 24(18), 2750-2756 (2006).

20. Muss HB, Berry DA, Cirrincione CT et al. Adjuvant chemotherapy in older women with early-stage breast cancer. N. Engl. J. Med. 360(20), 2055-2065 (2009).

- Highlights that chemotherapy is still of significant benefit in regards to recurrence risk and mortality in elderly patients, although toxicity may be greater.

21. Muss HB, Polley MC, Berry DA et al. Randomized trial of standard adjuvant chemotherapy regimens versus capecitabine in older women with early breast cancer: 10-year update of the CALGB 49907 trial. J. Clin. Oncol. 37(26), 2338-2348 (2019).

22. Kornblith AB, Lan L, Archer L et al. Quality of life of older patients with early-stage breast cancer receiving adjuvant chemotherapy: a companion study to cancer and leukemia group B 49907. J. Clin. Oncol. 29(8), 1022-1028 (2011).

23. Muss HB, Berry DA, Cirrincione $\mathrm{C}$ et al. Toxicity of older and younger patients treated with adjuvant chemotherapy for node-positive breast cancer: the Cancer and Leukemia Group B experience. J. Clin. Oncol. 25(24), 3699-3704 (2007).

24. Kizy S, Altman AM, Marmor $S$ et al. 21-gene recurrence score testing in the older population with estrogen receptor-positive breast cancer. J. Geriatr. Oncol. 10(2), 322-329 (2019).

25. Hurria A, Togawa K, Mohile SG et al. Predicting chemotherapy toxicity in older adults with cancer: a prospective multicenter study. J. Clin. Oncol. 29(25), 3457-3465 (2011).

26. Davies C, Godwin J, Gray R et al. Relevance of breast cancer hormone receptors and other factors to the efficacy of adjuvant tamoxifen: patient-level meta-analysis of randomised trials. Lancet 378(9793), 771-784 (2011).

27. Baum M, Budzar AU, Cuzick J et al. Anastrozole alone or in combination with tamoxifen versus tamoxifen alone for adjuvant treatment of postmenopausal women with early breast cancer: first results of the ATAC randomised trial. Lancet 359(9324), 2131-2139 (2002). 
28. Burstein HJ, Temin S, Anderson H et al. Adjuvant endocrine therapy for women with hormone receptor-positive breast cancer: American Society of Clinical Oncology clinical practice guideline focused update. J. Clin. Oncol. 32(21), 2255-2269 (2014).

29. Carlson RW, Moench S, Hurria A et al. NCCN task force report: breast cancer in the older woman. J. Natl Compr. Canc. Netw. 6(Suppl. 4), S1-S25 (2008).

- Outlines current areas of consensus and continued controversy of management in elderly patients.

30. Yourman LC, Lee SJ, Schonberg MA, Widera EW, Smith AK. Prognostic indices for older adults: a systematic review. JAMA 307(2), 182-192 (2012).

31. University of California San Francisco. ePrognosis (2020). https://eprognosis.ucsf.edu/ 